\title{
Preliminary study of an electro-mechanical artificial insemination device for small ruminants
}

\begin{abstract}
Artificial Insemination (AI) technique is used worldwide in goats breeding, especially in intensive systems reproduction control. AI is a low cost technique and simple to perform, however the labor cost is steep since the need experts are necessity. The immediate need to produce good profits in goats production, has initiated a development and initialization of an electro-mechanical artificial insemination device prototype for goats is initially developed. This device is able to increase the accuracy of semen deposited to the cervix by visualizing the os. This is realized by a built-in circuit utilizing a PIC as its brain and functions well as pre-programmed in the PIC. Furthermore, a continuous use of this device could significantly reduce the labor cost. However, since the operation time is relatively slow, further improvements are required.
\end{abstract}

Keyword: Artificial Insemination (AI); Reproduction; Electromechanical device; Goat 